\title{
On certain Quadric Hypersurfaces in Riemannian Space
}

\author{
By C. E. Weatherburs (Western Australia). \\ (Received 8th October, 1934. Read 2nd November, 1934.)
}

\section{The Hypersurfaces Defined.}

The use of geodesic polar coordinates in the intrinsic geometry of a surface leads to the concept of a geodesic circle, i.e. the locus of points at a constant distance from the pole $O$ along the geodesics through $O$. A geodesic hypersphere is the obvious generalisation of this for a Riemannian $V_{n}$. We propose to consider more general central quadric hypersurfaces of $V_{n}$, which we define as follows. Let $x^{i}(i=1,2, \ldots, n)$ be a system of coordinates in $V_{n}$, whose metric is $g_{i j} d x^{i} d x^{j}$, and let $a_{i j}$ be the components in the $x^{\prime}$ s of a symmetric covariant tensor of the second order, evaluated at the point $O$, which is taken as pole. If $s$ is the are-length of a geodesic through $O$, the quantities $\xi^{i}$ defined by

$$
\xi^{i}=\left(d x^{i} / d s\right)_{0}
$$

are the contravariant components of the unit vector in the direction of the geodesic at $O$, the suffix zero indicating that the derivative is to be evaluated at the pole. If $s$ is measured from $O$ along the geodesic to the current point $P$, the variables $y^{i}$ defined by

$$
y^{i}=\xi^{i} s
$$

are the Riemannian coordinates of $P$ relative to the pole $O$.

The quadric hypersurface defined by the equation

$$
y^{i} a_{i j} y^{j}=1
$$

is clearly a central quadric. For the equation may be expressed

$$
\xi^{i} a_{i j} \xi^{j}=1 / s^{2}
$$

showing that, on a given geodesic through $O$, there are two points of the hypersurface, in opposite directions along the geodesic, and at equal geodesic distances from the pole. The positive value of $s$ given by (2) may be called the geodesic radius of the quadric (1) for the 
direction $\xi^{i}$ at $O$. The particular case of a hypersphere, of geodesic radius $c$, corresponds to

$$
a_{i j}=g_{i j} / c^{2}
$$

For, if this value of $a_{i j}$ be substituted in (2), we obtain $s^{2}=c^{2}$, as required.

Further, we deduce immediately from (2) that

The sum of the inverse squares of the geodesic radii for $n$ mutually orthogonal directions at $O$ is an invariant, equal to $a_{i j} g^{i j}$.

For, if $e_{h 1}^{i}(h=1, \ldots, n)$ are the contravariant components of the unit tangents at $O$ to the curves of an orthogonal ennuple in $V_{n}$, it follows from (2) that the sum of the inverse squares of the geodesic radii for these directions is given by

as stated.

$$
\sum_{h}\left(s_{h \mid}\right)^{-2}=\sum_{h} e_{h \mid}^{i} a_{i j} e_{h \mid}^{j}=a_{i j} g^{i j}
$$

Let $y^{i}$ be the Riemannian coordinates of a point $P$, not necessarily on the quadric (1). Then the equation

$$
Y^{i} a_{i j} y^{j}=1
$$

defines a hypersurface of $V_{n}$, the quantities $Y^{i}$ being Riemannian coordinates of the current point on the hypersurface. We shall call this the polar hypersurface of $P$ relative to the quadric (1). If $P$ lies on the quadric it also lies on its polar hypersurface. It is easy to see that

The geodesic through $P$ and the centre of the quadric is divided harmonically by the quadric, the point $P$ and its polar hypersurface.

For if $\xi^{i}$ is the unit tangent to this geodesic at $O, s^{\prime}$ the geodesic distance of $P$ from $O$, and $s, s^{\prime \prime}$ those of the points $B$ and $Q$ in which the geodesic cuts the quadric and the polar hypersurface, the Riemannian coordinates of $Q$ are $\xi^{i} s^{\prime \prime}$; and since this point lies on (3) we have

$$
s^{\prime} s^{\prime \prime}\left(\xi^{i} a_{i j} \xi^{j}\right)=1
$$

and therefore, in virtue of (2),

as required.

$$
s^{\prime} s^{\prime \prime}=s^{2}
$$

Because the tensor $a_{i j}$ is symmetric, it follows from (3) that if the polar hypersurface of $P$ passes through a point $Q$, then that of $Q$ passes through $P$. 


\section{RectProcal QUadrics.}

Let $a^{i j}$ be the symmetric contravariant tensor at $O$ reciprocal to $a_{i j}$, so that

$$
a^{i j} a_{j k}=\delta_{k}^{i} .
$$

Let $\eta_{i}$ be the covariant components of the unit tangent to a geodesic at $O$, and $t$ the distance along this geodesic to a point $R$. If we write

$$
z_{i}=\eta_{i} t
$$

the quantities $z_{i}$ are covariant components of a vector at $O$,

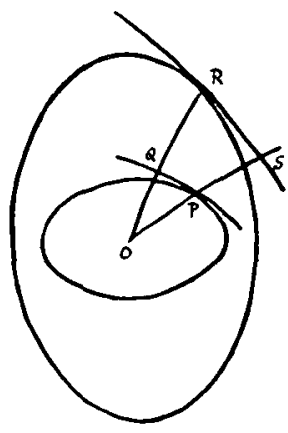

determining the point $R$ by its geodesic distance from the pole and the direction of the geodesic at $O$. Points $R$ which satisfy the relation

$$
z_{i} a^{i j} z_{j}=1
$$

also lie on a central quadric with centre at $O$. We shall call this the reciprocal quadric to (1).

Let $P$ be a point on (1) whose Riemannian coordinates $y^{i}$ are $\xi^{i} s$. Then the point whose coordinates $z_{i}$ are equal to $a_{i j} y^{j}$ lies on the reciprocal quadric. For, if these quantities are substituted for $z_{i}$ in (7), the equation is satisfied in virtue of (5). Let $R$ be this point on (7). The relation is reciprocal; for

$$
a^{i j} z_{j}=a^{i j} a_{j k} y^{k}=\delta_{k}^{i} y^{k}=y^{i},
$$

and it follows from (7) that

$$
z_{i} y^{i}=1
$$

so that, if $\theta$ is the inclination at $O$ of the central geodesics to $P$ and $R$,

$$
\text { st } \cos \theta=1 \text {. }
$$


Let $Q$ be the point in which the geodesic $O R$ cuts the polar hypersurface of $P$ with respect to (1), and $t^{\prime}$ the geodesic distance $O Q$. Then the Riemannian coordinates of $Q$ are $t^{\prime} \eta^{i}$; and since this point lies on the polar hypersurface (3), we have

that is

$$
t^{\prime} \eta^{i} a_{i j} y^{j}=1,
$$

$$
t^{\prime} \eta^{i} z_{i}=1
$$

and therefore, in virtue of (6),

$$
t t^{\prime}=1,
$$

since $\eta^{i}$ and $\eta_{i}$ are components of a unit vector. Thus the geodesic distances $O Q$ and $O R$ are reciprocal. Similarly if the geodesic $O P$ cuts the polar hypersurface of $R$ with respect to (7) in the point $S$ at a geodesic distance $s^{\prime}$ from $O$, the (covariant) coordinates of $S$ are $\xi_{i} s^{\prime}$; and since this point lies on the polar hypersurface

it follows that

$$
Z_{i} a^{i j} z_{j}=1 \text {, }
$$

which may be expressed

$$
s^{\prime} \xi_{i} y^{i}=1,
$$

$$
s \mathcal{s}^{\prime} \xi_{i} \xi^{i}=1 \text {. }
$$

Consequently

$$
s s^{\prime}=1
$$

and the geodesic distances $O P$ and $O S$ are reciprocal. Also from $\left(8^{\prime}\right),(9)$ and (10) it follows that

and

$$
s \cos \theta=t^{\prime}
$$

$$
t \cos \theta=s^{\prime} .
$$

It is easy to show that the hypersurface $Y^{i} \eta_{i}=q$ is the polar hypersurface of a point on (1) with respect to that quadric provided that

$$
\eta_{i} a^{i j} \eta_{j}=q^{2} ;
$$

and hence that the polar hypersurfaces of $n$ points $y_{h 1}^{i}$ on (1), for which the vectors $a_{i j} y_{h \mid}^{j}$ are mutually orthogonal, meet on the geodesic hypersphere $s^{2}=a^{i j} g_{i j}$.

\section{Conjugate Geodesic Radir.}

Before considering conjugate directions and radii of the quadric (1), we introduce the symmetric covariant tensor $A_{i j}$, evaluated at $O$ and defined by the relation

$$
A_{i k} g^{k l} A_{l j}=a_{i j} .
$$


Then, if $y^{i}$ are the Riemannian coordinates of the point $P$ on the quadric (1), this quadric is given by

Consequently

$$
y^{i} A_{i k} g^{k l} A_{l j} y^{j}=1 \text {. }
$$

where we have written

$$
E_{k} g^{k l} E_{l}=1,
$$

$$
E_{k}=y^{i} A_{i k} \text {. }
$$

From (13) it is evident that these quantities $E_{k}$ are covariant components of a unit vector, whose contravariant components $E^{i}$ are therefore given by

$$
E^{i}=g^{i j} \dot{E}_{j}=g^{i j} A_{j k} y^{k} .
$$

The components $z_{i}$ of the covariant vector defining the corresponding point $R$ on the reciprocal quadric are

$$
z_{i}=a_{i j} y^{j}=A_{i k} g^{k l} A_{l j} y^{j}=A_{i k} E^{k} .
$$

If $A^{i j}$ is the reciprocal contravariant tensor to $A_{i j}$, the relations (14) and (15) are equivalent to

and

$$
\begin{aligned}
& y^{i}=A^{i k} E_{k} \\
& E^{i}=A^{i j} z_{j} .
\end{aligned}
$$

And it is easily verified that, in terms of this tensor,

$$
A^{i k} g_{k l} A^{l j}=a^{i j} \text {. }
$$

The directions of the vectors $u^{i}$ and $v^{i}$ at $O$ will be said to be conjugate with respect to the quadric (1) when they satisfy the relation

$$
u^{i} a_{i j} v^{i}=0
$$

and the geodesics which pass through $O$ in these directions will be called conjugate geodesic diameters of (1). If $y_{h \mid}^{i}$ and $y_{k \mid}^{i}$ are the Riemannian coordinates of the extremities of conjugate geodesic diameters, we deduce from (17) and (14) that

that is

$$
y_{h \mid}^{i} A_{i l} g^{l p} A_{p j} y_{k \mid}^{j}=0 ;
$$

$$
E_{h \mid l} g^{l p} E_{k \mid p}=0,
$$

showing that the corresponding vectors $E_{h !}^{i}$ and $E_{k \mid}^{i}$ are orthogonal. It is easily verified that, if the conditions (18) are satisfied, the corresponding points $z_{h \mid i}$ on (7) are the extremities of mutually conjugate geodesic radii of that quadric.

We may now establish the theorem: 
The sum of the squares of $n$ mutually conjugate geodesic radii of the quadric (1) is an invariant, equal to $a^{i j} g_{i j}$.

Let $y_{h \mid}^{i}(h=1, \ldots, n)$ be the Riemannian coordinates of the extremities of the $n$ mutually conjugate geodesic radii, and $E_{h \mid i}$ the corresponding unit vectors (14). The conjugate relations are expressed by (18), where $h \neq k$. Further, if $s_{h}$ is the length of the geodesic radius to $y_{h \mid}^{i}$, and $\xi_{h \mid}^{i}$ its direction at $O$, it follows from (14') that

$$
s_{h \mid} \xi_{h \mid}^{i}=A^{i j} E_{h \mid j} .
$$

Taking the square of the length of each member, and summing for $h$ from 1 to $n$, we have

$$
\begin{aligned}
\sum_{h}\left(s_{h \mid}\right)^{2} & =\sum_{h} E_{h \mid j} A^{j i} g_{i p} A^{p q} E_{h \mid q} \\
& =\sum_{h} E_{h \mid j} a^{j q} E_{h \mid q}=a^{j q} g_{j q},
\end{aligned}
$$

since the $n$ vectors $E_{h \mid i}$ are mutually orthogonal unit vectors.

We may also observe in passing that

The polar hypersurfaces of the extremities of $n$ mutually conjugate geodesic radii of the quadric (1) meet on a similar quadric.

For, in virtue of (3), (12) and (14), the polar hypersurface of the extremity $y_{h \mid}^{i}$ is given by

$$
Y^{i} A_{i l} g^{l k} E_{h \mid k}=1 .
$$

Squaring both members, and summing for $h$ from 1 to $n$ we obtain

and therefore

$$
\underset{h}{\Sigma}\left(Y^{i} A_{i l} g^{l k} E_{h \mid k}\right)\left(E_{h \mid q} g^{q p} A_{p j} Y^{j}\right)=n,
$$

$$
Y^{i} A_{i l} g^{l k} g_{k q} q^{q p} A_{p j} Y^{j}=n,
$$

which, in virtue of $(12)$, reduces to

$$
Y^{i} a_{i j} Y^{j}=n \text {. }
$$

Thus the locus of the intersection $Y^{i}$ of the $n$ polar hypersurfaces is a similar quadric.

\section{Application.}

Let the $V_{n}$ considered above be a hypersurface of an enveloping Riemannian $V_{n+1}$, with $\Omega_{i j} d x^{i} d x^{j}$ as the second fundamental form of $V_{n}$. It is well known that the quantities $\Omega_{i j}$ are components of a 
symmetric covariant tensor ${ }^{1}$, and that the normal curvature $\kappa_{n}$ of the hypersurface for the direction of the unit vector $\xi^{i}$ is given by

$$
\kappa_{n}=\xi^{i} \Omega_{i j} \xi^{j} .
$$

It follows that, with the same notation as above, the normal curvature is equal to the inverse square of the geodesic radius of the quadric

$$
y^{i} \Omega_{i j} y^{j}=1
$$

in the direction $\xi^{i}$ at $O$. And from the first theorem of $\S 1$ we then deduce that

The sum of the normal curvatures of the hypersurface $V_{n}$ for $n$ mutually orthogonal directions at a point is invariant, and equal to $\Omega_{i j} g^{i j}$.

Similarly from the first theorem of $\S 3$ it follows that, if $\Omega^{i j}$ is the reciprocal tensor to $\Omega_{i j}$,

The sum of the reciprocals of the normal curvatures of the hypersurface $V_{n}$ for $n$ mutually conjugate directions at a point of it is invariant, and equal to $\Omega^{i j} g_{i j}$.

The quadric (21) corresponds to Dupin's indicatrix for a surface.

1 Cf. Eisenhart, Riemannian Geometry, $\$ \$ 43,44$. 